\title{
COHERENT SYNCHROTRON RADIATION IN AN X-BAND PHOTOINJECTOR
}

\author{
F.V. Hartemann, D. J. Gibson, LLNL, Livermore, CA 94550, USA \\ A. L. Troha, University of California, Davis, CA 95616, USA
}

\section{Abstract}

A high-gradient, high-brightness X-band rf photoinjector is used to produce sub-picosecond electron bunches at energies in the $1.5-2 \mathrm{MeV}$ range, which are propagated in a short section of corrugated waveguide, with an inner diameter of $10 \mathrm{~mm}$, a corrugation period of approximately $2.5 \mathrm{~mm}$, and a modulation depth of 1.5 $\mathrm{mm}$. Extremely short rf pulses are measured, in the $100 \mathrm{ps}$ $-<5$ ns range, corresponding to the slippage time between the electron bunch and the radiation produced by a coupling to the slow-waves supported by the waveguide structure. Coherence is measured by varying the bunch charge and verifying a quadratic scaling of the rf power; $\mathrm{W}$-band radiation is produced using this new radiation mechanism, which may have very important potential applications in coherent millimeter-wave and $\mathrm{THz}$ radiation generation. We will discuss the technical details of the X-band rf gun and slow-wave circuit, as well as our experimental results, and a theoretical description of the interaction. A proposed millimeter-wave, chirped-pulse free-electron laser experiment using the X-band rf gun will also be discussed.

\section{EXPERIMENTAL SET-UP}

The experimental set-up used is shown in Fig. 1. A complete discussion of the equipment is given in [1]; it shall be summarized here only as it relates to the coherent synchrotron radiation measurements. There are two interconnected systems necessary to produce the photoelectron beam: the laser system needed to produce the electrons, and the $\mathrm{x}$-band $\mathrm{rf}$ system needed to

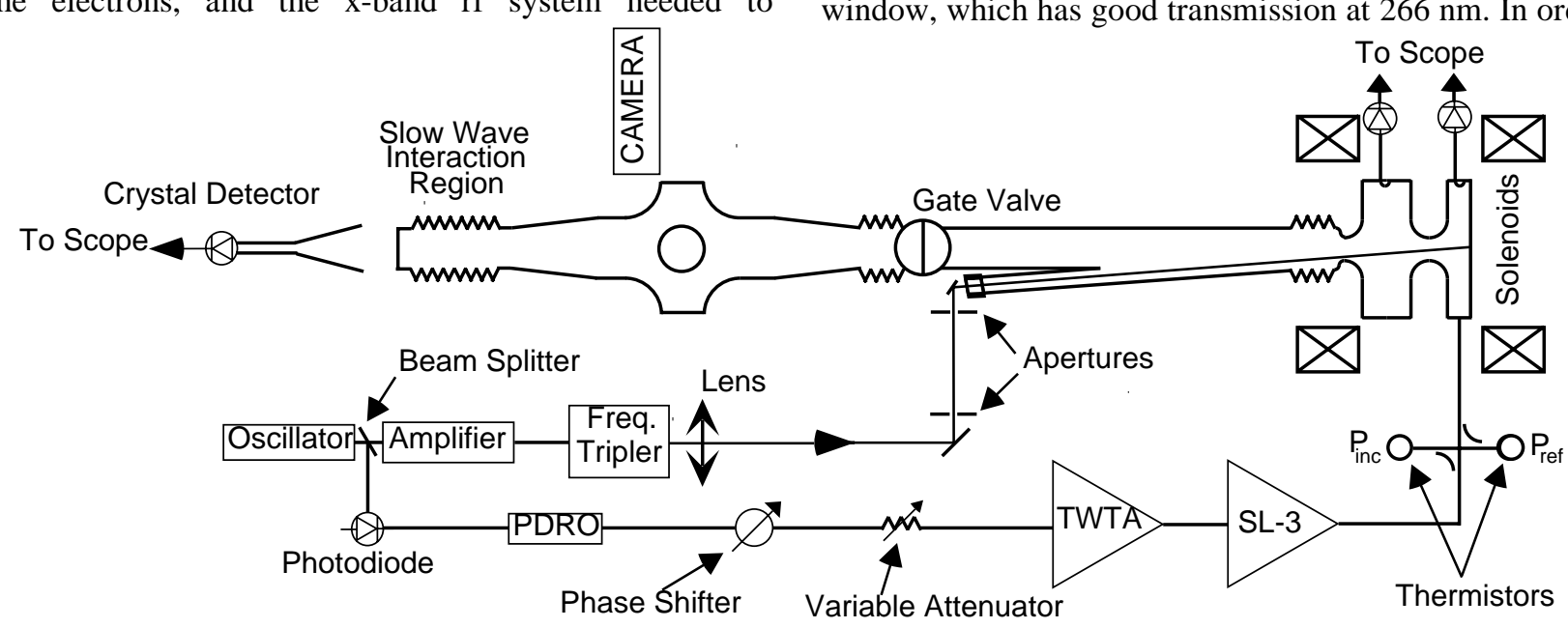

Figure 1: Experimental Setup accelerate them. The laser system used to illuminate the photocathode is a commercial Titanium:Sapphire ( $\mathrm{Ti}: \mathrm{Al}_{2} \mathrm{O}_{3}$ )-based chirped-pulse amplification (CPA) system, consisting of an oscillator, a grating stretcher, a regenerative amplifier, a grating compressor, and a frequency tripler. The system is based entirely on diode pumped solid-state laser (DPSSL) technology, which provides the stability needed to illuminate the photocathode reliably. The oscillator is pumped by a $5 \mathrm{~W}$ $\mathrm{CW}, 532 \mathrm{~nm}$ beam and produces a pulse that is $15 \mathrm{fs}$ in duration with a bandwidth of $>70 \mathrm{~nm}$ centered around $800 \mathrm{~nm}$ at a repetition rate of $79.138 \mathrm{MHz}$. The output of the oscillator is then coupled into a sub-50 fs, $1 \mathrm{kHz}$ Spitfire system from Positive Light, which includes the grating stretcher, $\mathrm{Ti}: \mathrm{Al}_{2} \mathrm{O}_{3}$ regenerative amplifier, and grating compressor. The regenerative amplifier crystal is pumped by a $7 \mathrm{~W}$-average power, $1 \mathrm{kHz}$, Q-switched laser and amplifies one pulse from the oscillator pulsetrain to an energy of $0.4 \mathrm{~mJ}$ with a duration of $60 \mathrm{fs}$ at an overall repetition rate of $1 \mathrm{kHz}$. The amplified pulse is then directed into a frequency tripler which yields $266 \mathrm{~nm}$ UV pulses with $6 \mu \mathrm{J}$ of energy. Due to fluctuations in the duration of the output pulse of the Spitfire, the intensity of the UV pulse varied between 5 and $7 \mu \mathrm{J}$ on a time scale of a few seconds. These fluctuations seem to be related to instabilities, e.g. from air currents, in the compressor section of the Spitfire.

The UV beam is injected onto the photocathode at 3 degrees from normal incidence using a custom-made high-vacuum "Y" piece attached to the output face of the gun. The straight arm of the "Y" is the photoelectron beamline, and the 3-degree arm is sealed with a Suprasil window, which has good transmission at $266 \mathrm{~nm}$. In order 

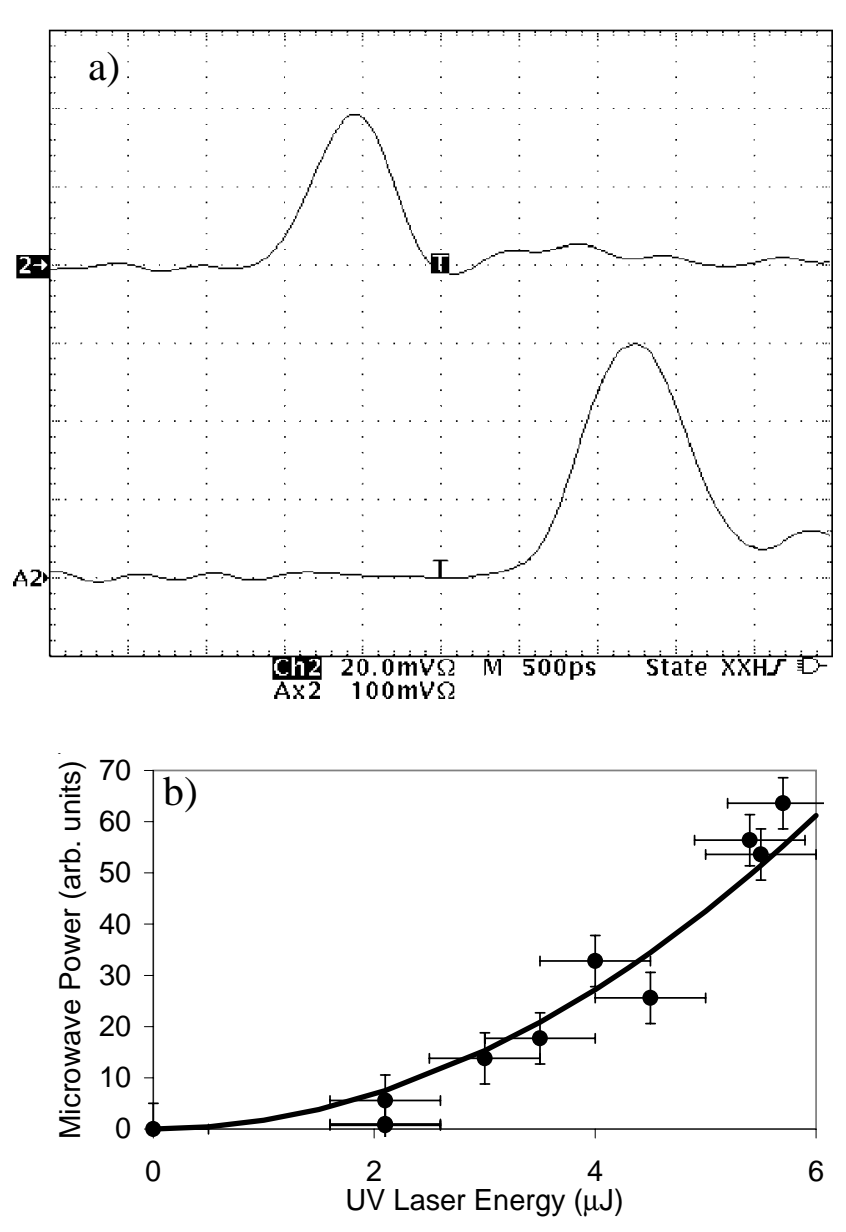

Figure 2: a) Upper trace: $x$-band detector signal, Lower trace: laser photodiode signal. Both are at the resolution of the detectors. b). Microwave power as a function of laser energy with a quadratic fit.

to align the laser down the beampipe properly, a lamp was placed against the beamline (where the camera is shown in Fig. 1) to illuminate the copper photocathode, which diffusely reflected some of the light down the "Y". Two apertures were then placed such that the cathode could clearly be seen through both of them, and the laser was subsequently passed through the apertures.

The second major component of the set-up is the rf system, which delivers $1 \mathrm{MW}$ of $\mathrm{X}$-band rf power to the gun. To ensure proper synchronization between the laser and $\mathrm{rf}$ systems, the $\mathrm{rf}$ is derived from a photodiode monitoring the $79.138 \mathrm{MHz}$ oscillator pulse-train mentioned above. This signal, after proper filtering, is sent to a phase-locked dielectric resonance oscillator (PDRO), which multiplies the frequency by a factor of 108 , producing the $8.547 \mathrm{GHz}$ signal used for the gun. This signal is sent through a phase shifter, which allows study of the effect of the timing of laser injection relative to the rf phase, and a variable attenuator, which allows for adjustment of the final gun power. It is then sent to a 1 $\mathrm{kW}$ travelling wave tube amplifier (TWTA), followed by a Stanford Linear Accelerator Center (SLAC) SL-3 Klystron which produces $250 \mathrm{~ns}$ pulses at $20 \mathrm{~Hz}$.

The heart of the rf system is a high- $Q$ (4,274 measured), $1-1 / 2$ cell, $X$-band $\mathrm{rf}$ gun with a custom tuning post piece was fabricated by SLAC to ensure that the peak of the gun resonance is properly correlated with the maximum of the SL-3 rf power tuning curve. The tuning of the rf gun coupling is supplemented by two independent tuners for the half and full cells, and the overall resonant frequency of the system can be temperature-tuned once balanced, $\pi$ mode critical coupling is achieved. Monitoring of the fields in the half and full cells to ensure proper operation is provided by magnetic pickup loops, which are designed with very low coupling parameters in order to avoid perturbing the rf fields in the accelerating structure and causing rf breakdown.

\section{BEAM PROPERTIES}

With proper alignment of the laser and timing and phasing of the rf pulse, a photoelectron beam is produced, which is discussed in detail in [1]. In order to minimize the dark current background in the beam, rf power of 1-2 MW was used in the gun giving beam energies between 1.4 and $2 \mathrm{MeV}$. The photoelectron beam is optimized by examining Faraday cup measurements and the image of the beam on a phosphor screen; adjustments can be performed by varying the laser injection phase, the rf power energizing the gun, or the solenoid focusing strength. This beam consists of $25 \mathrm{pC}$ electron bunches with an energy of $1.47 \mathrm{MeV}$ (depending on the rf power). Electron spectrometer measurements show the beam to have an energy spread of $\Delta \gamma / \gamma_{0}<1.8 \%$ FWHM, and beam divergence measurements show the normalized emittance to be $\varepsilon_{n} \approx 1.65 \pi \mathrm{mmmrad}$. Measurements taken with a streak camera show that the electron bunch duration must be below the 2 ps resolution of the camera. This generated beam is observed to have a fairly large amount of random fluctuation. It is believed that most of this is due to instabilities in the intensity and pointing of the laser system. In measuring the beam parameters above, single-shot measurements were performed to capture some of the cleaner events; therefore these numbers represent an optimal beam which was not continuously generated. The exception is the bunch charge measurement, which results from a $5 \mathrm{~s}$ average over 100 shots.

\section{COHERENT SYNCHROTRON RADIATION MEASUREMENTS}

The experimental measurements to study coherent synchrotron radiation consisted of propagating photoelectron bunches through a corrugated waveguide so they could couple to the slow waves supported by that structure. The rf power radiated by the bunches is measured with a coupling horn, a waveguide section, and various detectors. The emission of extremely short pulses of rf power up to Ka-band was confirmed; at higher 

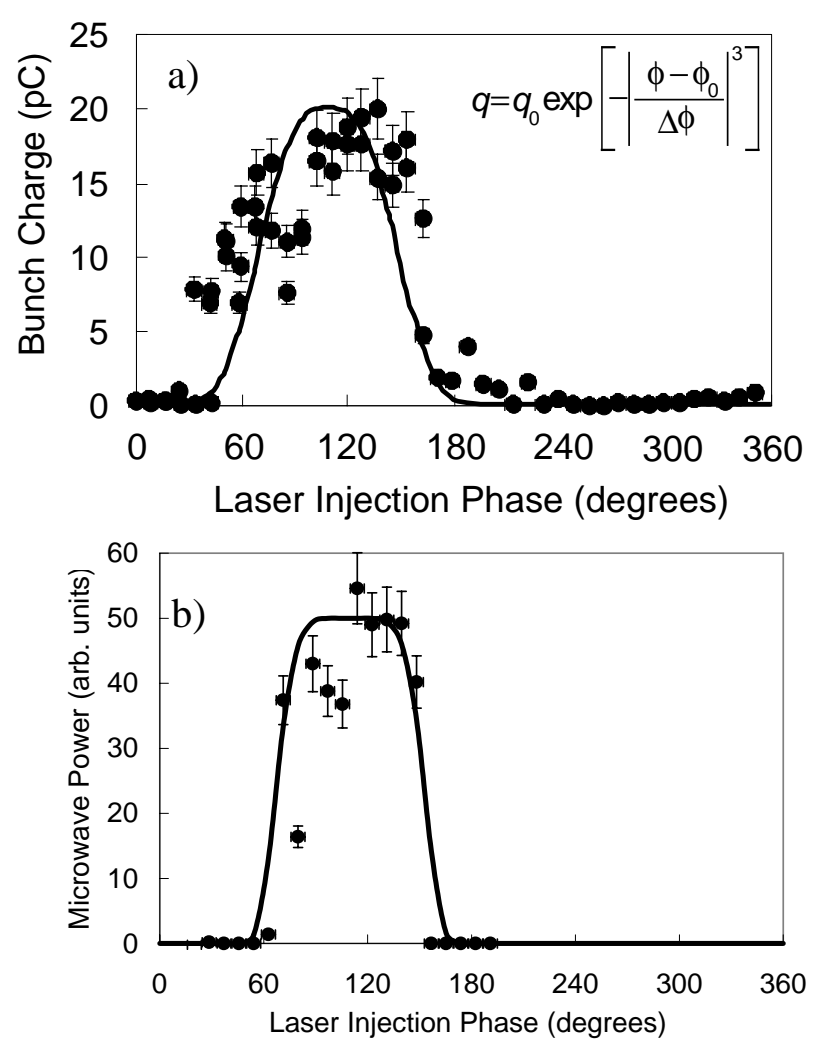

Figure 3: a) Measured beam charge as a function of injection phase, with fit shown. b) Measured

microwave power as function of laser injection phase with the same fit as (a), squared and scaled in amplitude.

frequencies, the detector speed is believed to be insufficient: the characteristic time-scale of the radiation bursts is given by the slippage between the photo-electron bunch and the radiation pulse; as the corrugated waveguide section was only $10 \mathrm{~cm}$ long, the pulses were estimated to be $50-500$ ps in duration, or approximately $20 \mathrm{rf}$ cycles. At low frequency, between X-band and Kaband, pulses with a FWHM of 500 ps were measured, as illustrated in Fig. 2(a); this is to be compared with the detector rise-time, specified at $<600$ ps. At higher frequencies, the detectors are not fast enough to follow the pulses, and we are considering the possibility of using broadband mixers to perform more detailed measurements.

To demonstrate that the radiation produced is, indeed, coherent, the power radiated as a function of the bunch charge is measured. There are two different ways to vary the bunch charge; the first is holding the laser injection phase fixed and adjusting the UV energy by using different combinations of calibrated neutral density filters, as shown in Fig. 2(b). For coherent radiation, the energy should vary as the square of the bunch charge, which varies linearly as the laser energy; a quadratic fit to the data is shown in Fig. 2(b). The good agreement indicates the presence of coherent synchrotron radiation.
The second method of varying the bunch charge is by holding the laser energy fixed and adjusting the laser injection phase. The $\mathrm{rf}$ phase relative to the laser was varied over 360 degrees; Variation of the charge extracted as a function of rf phase for a fixed UV intensity is shown, along with a supergaussian, in Fig. 3(a). Making the same variation of phase while recording instead the microwave power results in the plot shown in Fig. 3(b). The supergaussian fit from Fig. 3(a) is squared and scaled to the units of Fig. 3(b) and also shown, giving excellent agreement with the data. This is further evidence for the production of coherent synchrotron radiation.

\section{CONCLUSIONS}

This result has important potential application to millimeter-wave and far infrared coherent radiation generation, as the electron beam produced by the compact rf photo-injector are fully pre-bunched, and can radiated coherently up to the $\mathrm{THz}$ spectral region. In particular, our group has produced extensive theoretical work on prebunched free-electron masers [2] and the theory of coherence in synchrotron radiation [3], and shown that ultra-wideband, chirped-pulse radiation could be produced at power levels in excess of $2 \mathrm{MW}$, in the 100$200 \mathrm{GHz}$ range. Such devices could be extremely useful for advanced radar systems, and as tools to probe materials and plasmas.

These measurements also indicate that one must pay very close attention to the beamline design for a system using this technology, as coherent synchrotron radiation typically degrades the high-brightness bunches because of correlated recoil effects. This implies that one must avoid bends, bellows, and cavities, which all strongly couple to the photoelectron bunches, and instead strive to provide smooth, continuous transverse boundaries for the beamline, in order to minimize unwanted radiation. Furthermore, the beamline can also be designed to avoid trapped modes, and to provide quenching of the lower frequency modes by a judicious combination of polarization and cut-off frequencies for those modes that couple most strongly with the pre-bunched beam.

\section{ACKNOWLEDGEMENTS}

This work was performed under the auspices of the U.S. Department of Energy by the University of California, Lawrence Livermore National Laboratory, partially through the Institute for Laser Science and Applications, under contract No. W-7405-Eng-48. Additional funding was provided by AFOSR Grant No. F4962099-1-0297 and NIH Contract No. N01-CO-97113.

\section{REFERENCES}

[1] D. J. Gibson, F. V. Hartemann, et. al, Phys. Rev. Special Topics: Accel. and Beams. (In Review)

[2] G. P. Le Sage, et. al., IEEE Trans. Plasma Sci. 24, p. 781 (1996).

[3] F. V. Hartemann, Phys. Rev. E 61, p. 972 (2000). 